\title{
Intensity-dependent effect of treadmill running on differentiation of rat bone marrow stromal cells
}

\author{
SHENG-YAO LIU ${ }^{1}$, ZHE LI $^{1}$, SHAO-YONG XU ${ }^{1}$, LEI XU ${ }^{1}$, MO YANG $^{2}$ and GUO-XIN NI ${ }^{1,3}$ \\ Departments of ${ }^{1}$ Orthopedics and Traumatology and ${ }^{2}$ Hematology, Nanfang Hospital, \\ Southern Medical University, Guangzhou, Guangdong 510515; ${ }^{3}$ Department of Rehabilitation Medicine, \\ First Affiliated Hospital, Fujian Medical University, Fuzhou, Fujian 350005, P.R. China
}

Received April 25, 2016; Accepted March 23, 2017

DOI: $10.3892 / \mathrm{mmr} .2018 .8797$

\begin{abstract}
The effect of running on bone mass depends on its intensity. However, the underlying molecular mechanism that associates running intensity with bone mass is unclear. The current study examined the effects of treadmill running at different intensities on bone mass and osteogenic differentiation of bone marrow stromal cells (BMSCs) in a rat model. A total of 24 male Wistar rats were randomly divided into groups and subjected to no running (Con group), low-intensity running (LIR group), moderate-intensity running (MIR group), and high-intensity running (HIR group). Histological, immunohistochemistry and micro-CT examinations were performed on the femora harvested after 8 weeks of treadmill running. The study demonstrated that treadmill running affected trabecular bone mass in an intensity-dependent manner. In addition, such an intensity-dependent effect was also demonstrated on the osteogenic and adipogenic differentiation and proliferation of BMSCs. Furthermore, the Wnt/ $\beta$-catenin signaling pathway may be involved in the running-induced increase in bone mass in rats in the MIR group. There appears to be a biomechanical 'window', in which running-induced strain signals can increase the number of BMSCs and progenitor cells (specific to the osteoblast lineage) causing upregulation of osteogenesis and downregulation of adipogenesis of BMSCs. This finding may provide insight into the molecular and cellular mechanisms responsible for bone homeostasis.
\end{abstract}

Correspondence to: Professor Guo-Xin Ni, Department of Orthopedics and Traumatology, Nanfang Hospital, Southern Medical University, 1838 Guangzhou Avenue, Guangzhou, Guangdong 510515, P.R. China

E-mail: fgxni@graduate.hku.hk

Professor Mo Yang, Department of Hematology, Nanfang Hospital, Southern Medical University, 1838 Guangzhou Avenue, Guangzhou, Guangdong 510515, P.R. China

E-mail: yangm1091@yahoo.com.hk

Key words: running, intensity, bone marrow stromal cells

\section{Introduction}

Running is one of the most common weight-bearing exercises and promotes general health and well-being $(1,2)$. However, the effect of running on bone mass is poorly understood. In a review, Iwamoto et al (3) demonstrated that treadmill running may increase bone mass in young and adult rats, particularly within the long bones at weight-bearing sites. However, Bourrin et al (4) reported that excessive treadmill running may cause the opposite effect on rat tibia. In parallel, the deleterious effects of endurance running on bone have also been reported in populations exposed to high-intensity exercise $(5,6)$. These inconsistent findings clearly imply that intensity may be an important factor influencing the effect of running on bone mass. However, the association between running intensity and bone mass is poorly understood.

It is well documented that, at the tissue level, bone adapts to changes in mechanical loading. Bone formation in healthy adults is mainly determined by the mechanical load history (7). In addition, animal models have demonstrated that bone remodeling is associated with a number of loading parameters, including the magnitude of the load $(8,9)$. The mechanisms involved in bone adaptation involve a multistep process of cellular mechanotransduction that stimulates bone remodeling resulting in either bone formation or resorption (10). However, the cellular basis whereby bone adapts to mechanical loads remains unclear (10). According to the mechanostat theory, bone mass adjusts when a typical load diverges from a physiological 'set-point', similar to a thermostat (7). The resident cells within bone tissue detect and subsequently respond to changes in the mechanical load. It is, therefore, hypothesized that treadmill running at different intensities would lead to different cellular responses, and, consequently, different bone adaptations.

Although osteocytes (which comprise $>90 \%$ of bone cells) are most likely responsible for sensing mechanical loads (11), evidence suggests that mechanical signals are influenced by the resident bone cell population and by their progenitors (12). Bone marrow stromal cells (BMSCs) are a type of self-renewing multipotential stem cell which can differentiate into a number of lineages, including osteoblasts, chondroblasts, and adipocytes (13). A body of evidence also suggests that bone adaptation is associated with either osteogenic or 
adipogenic differentiation of BMSCs (12,14-17). Specifically, mechanical stimuli may have an important role in influencing the osteogenic differentiation process of BMSCs and their production of mineralized matrix in vivo and in vitro (18). In addition, increased adipogenesis was observed at the expense of osteogenesis under conditions of hind limb unloading (19) and joint immobilization (20), whereas, mechanical loading (21) and climbing exercises (22), upregulated osteogenesis and downregulated adipogenesis of BMSCs. However, the method whereby BMSCs respond to mechanical signals induced by treadmill running remains unclear, and, in particular, how such a response is associated with to running intensity.

It is thought that cartilage and bone may respond to mechanical loading in an intensity-dependent manner (23). Using a rat model, we previously demonstrated that treadmill running with low-to-moderate intensity maintains cartilage homeostasis, whereas, high-intensity running may cause cartilage degradation (24). In the present study, the same animal model was used to examine the effect of different intensities of treadmill running on bone mass. To further gain insight into the mechanisms responsible for the differentiation potential of BMSCs, both osteogenic and adipogenic lineages were investigated under various mechanical loading conditions.

\section{Materials and methods}

Experimental animals and exercise protocols. This study was approved by the animal ethics committee of Nanfang Hospital, Southern Medical University (Guangzhou, China). The methods were performed out in accordance with the approved guidelines. A total of 24 specific pathogen-free grade adult male Wistar rats (13-14 weeks old, 180-220 g) were randomized into four even groups ( $\mathrm{n}=6$ per group) as follows: Control (CON); low-intensity running (LIR); moderate-intensity running (MIR); and high-intensity running (HIR). All animals were housed in cages with a temperature of $22 \pm 1^{\circ} \mathrm{C}, 40-70 \%$ humidity and a controlled light/dark cycle of 12/12 h. Furthermore, food and water were provided ad libitum. Animals in the Con group maintained a sedentary lifestyle. Those in LIR, MIR, and HIR groups were subjected to treadmill running according to the running protocols described previously (24). The constant speed and inclination varied as follows: LIR, $15.2 \mathrm{~m} / \mathrm{min}$ with $0^{\circ}$ incline for $60 \mathrm{~min}, 5$ days/week; MIR, $19.3 \mathrm{~m} / \mathrm{min}$ with $5^{\circ}$ incline for $60 \mathrm{~min}, 5$ days/week; and HIR, $26.8 \mathrm{~m} / \mathrm{min}$ with $10^{\circ}$ incline for $60 \mathrm{~min}, 5$ days/week.

After 8 weeks, all animals were sacrificed under anesthesia by cervical dislocation. Bilateral femora from each animal were dissected free of soft tissues and maintained in cold phosphate-buffered saline (PBS) on ice. The bones were cut open at both ends and bone marrow was collected from their central shafts. The distal end of the left femur from each rat was fixed in $10 \%$ formalin for histological and immunohistochemistry examinations, while the distal end of the right femur was used for micro-computed tomography (CT) scanning.

Histological and immunohistochemistry examinations. Following dissection of the distal end of the left femur from each rat, it was immediately immersed in fixative solution ( $10 \%$ formalin at $\mathrm{pH} 7.4$ ) for $24 \mathrm{~h}$ for histological, morphological and immunohistochemistry examinations. Decalcification was accomplished in $10 \%$ ethylenediaminetetraacetic acid solution prior to embedding of the samples in paraffin wax. They were then transversally sectioned in the diaphyseal region at a thickness of $5 \mu \mathrm{m}$.

To assess adipocyte density, sections were stained with hematoxylin and eosin (H\&E). Density of adipocytes (adipocyte number per $\mathrm{mm}^{2}$ marrow area, excluding trabeculae) was measured and quantified with image analysis software (Nikon H600L microscope and image analysis system; Nikon Corporation, Tokyo, Japan) by calculating the mean value of three sequential images from each of the six animal specimens. $\beta$-catenin was immunostained using the two-step immunohistochemistry method as previously described (25). The areas of interest (AOI) were selected via the irregular AOI tools using Image-Pro Plus 6.0 software (Media Cybernetics, Inc., Rockville, MD, USA). The ratio of integrated optical density (IOD) to area in each region was also calculated using Image-Pro Plus 6.0 software (Media Cybernetics, Inc.), and subsequently averaged for $\beta$-catenin content in each section.

Micro-CT measurements. Within $\sim 1$ week after fixation, the distal ends of the right femurs were scanned using a micro-CT scanner (SkyScan 1076 Micro-CT system; Bruker Corporation, Billerica, MA, USA). The scanner was set at a voltage $88 \mathrm{kV}$, a current of $100 \mu \mathrm{A}$ and a resolution of $18 \mu \mathrm{m}$ per size. The region of interest was the area $1.0 \mathrm{~mm}$ below the lower end of the growth plate extending $3.2 \mathrm{~mm}$ distally. A global threshold 90 to 255 was used for all samples to identify mineralized tissue/soft tissue. For analysis of trabecular bone, a cube of trabecular bone with a size of $1.04 \times 1.04 \times 1.04 \mathrm{~mm}^{3}$ in the ROI was selected. Three-dimensional structural parameters measured included trabecular bone volume (BV/TV), bone mineral density (BMD), trabecular number (Tb.N), trabecular thickness (Tb.Th), trabecular separation (Tb.Sp), structure model index (SMI) and the degree of anisotropy (DA).

Rat BMSCs cultures. Immediately after the distal ends of both femora were excised, the bone marrow was flushed into a $15 \mathrm{ml}$ sterile centrifuge tube using L-Dulbecco's modified Eagle's medium (L-DMEM; Gibco; Thermo Fisher Scientific, Inc., Waltham, MA, USA) containing 10\% fetal bovine serum stromal medium. The marrow isolate was centrifuged at $179 \mathrm{x} \mathrm{g}$ for $5 \mathrm{~min}$ at room temperature, and the pellet was resuspended in $4 \mathrm{ml}$ L-DMEM supplemented with $10 \%$ fetal bovine serum (FBS; Gibco; Thermo Fisher Scientific, Inc.).

Colony-forming unit (CFU) assay. For CFU-fibroblast (CFU-F) assay, bone marrow femora were flushed into L-DMEM and centrifuged at $179 \mathrm{xg}$ for $5 \mathrm{~min}$ at room temperature. The medium was then suspended in L-DMEM supplemented with 10\% FBS (Gibco; Thermo Fisher Scientific, Inc.). Following 7 days of incubation at $37^{\circ} \mathrm{C}$, non-adherent cells were removed by rinsing with PBS. Adherent stromal cells were plated on a $35 \mathrm{~mm}$ sterile culture dish ( $2 \times 10^{6}$ cells/dish) and incubated in L-DMEM supplemented with 10\% FBS (Gibco; Thermo Fisher Scientific, Inc.). At day 10, the cultures were fixed with $4 \%$ paraformaldehyde for $20 \mathrm{~min}$ at room temperature and stained with $0.1 \%$ crystal violet for $30 \mathrm{~min}$. The number of colonies was counted under a light microscope. 
Table I. Primer sequences used for reverse transcription-quantitative polymerase chain reaction.

\begin{tabular}{lllr} 
Gene & \multicolumn{1}{c}{ Forward } & \multicolumn{1}{c}{ Reverse } & $\begin{array}{r}\text { Product } \\
\text { size (bp) }\end{array}$ \\
\hline GAPDH & 5'-GGCACAGTCAAGGCTGAGAATG-3' & 5'-ATGGTGGTGAAGACGCCAGTA-3' & 143 \\
RUNX2 & 5'-GGCCACTTACCACAGAGCTA-3' & 5'-GAGGCGGTCAGAGAACAAAC-3' & 109 \\
SP7 & 5'-GTCCTCTCTGCTTGAGGAAG-3' & 5'-CTGTTGAGTCTCGCAGAGG-3' & 107 \\
Collagen I & 5'-CATGTTCAGCTTTGTGGACC-3' & 5'-TTAGGGACCCTTAGGCCATT-3' & 120 \\
ALP & 5'-AACAACCTGACTGACCCTTC-3' & 5'-TCCACTAGCAAGAAGAAGCC-3' & 92 \\
PPAR $\gamma 2$ & 5'-GATCCTCCTGTTGACCCAGA-3' & 5'-CTGATTCCGAAGTTGGTGGG-3' & 119 \\
$\beta$-catenin & 5'-TTGTACGAGCACATCAGGAC-3' & 5'-GCACCCTTCAACTATCTCCTC-3' & 101 \\
Osteocalcin & 5'-GACTGCATTCTGCCTCTCTG-3' & 5'-ATTCACCACCTTACTGCCCT-3' & 100
\end{tabular}

RUNX2, runt related transcription factor 2; Sp7, Sp7 transcription factor; ALP, alkaline phosphatase; PPAR $\gamma 2$, peroxisome proliferator activated receptor $\gamma 2$.

For CFU-osteoblast (CFU-Ob) assay, BMSCs were initially cultured in stromal medium. After 7 days, cells were cultured in osteogenic induction medium consisting of DMEM, $10 \%$ FBS, $20 \mathrm{mmol} / \mathrm{l}$ dexamethasone, $10 \mathrm{mmol} / 1 \beta$-glycerophosphate and $50 \mu \mathrm{g} / \mathrm{ml}$ sodium 2-phosphate ascorbate. At day 14, the plates were stained with an alkaline phosphatase (ALP) staining kit (Nanjing Jiancheng Bioengineering Institute, Nanjing, China) for $30 \mathrm{~min}$.

Cell proliferation assay. For a proliferation assay, cells were cultured in 96-well plates at a concentration of $5 \times 10^{4}$ cells/well for 10 days. The culture medium (L-DMEM supplemented with 10\% FBS) was changed every 2-3 days. Cell proliferation was detected using methyl thiazolyl tetrazolium (MTT) assay. Briefly, $20 \mu 1$ MTT (5 mg/ml; Sigma-Aldrich; Merck KGaA, Darmstadt, Germany) was added to each well for $4 \mathrm{~h}$ at $37^{\circ} \mathrm{C}$. Subsequently, the supernatant was replaced with $150 \mathrm{ml}$ DMSO. After $15 \mathrm{~min}$ oscillation, the optical density (OD) was quantified at $490 \mathrm{~nm}$ using a SpectraMAX M2 microplate reader.

Osteogenic differentiation. Cells were plated at $5 \times 10^{5}$ cells $/$ well in 12-well plates and cultured in DMEM (Sigma-Aldrich; Merck KGaA). At day 10, the medium was replaced with fresh osteogenic induction medium containing $10 \mathrm{mmol} / \mathrm{l}$ $\beta$-glycerophosphate, $50 \mu \mathrm{g} / \mathrm{ml}$ sodium 2-phosphate ascorbate, $20 \mathrm{mmol} / 1$ dexamethasone (Sigma-Aldrich; Merck KGaA). Medium was changed every 3 days. Osteogenic differentiation of BMSCs was assessed on day 14 using ALP activity assay and staining, and on day 21 using Alizarin red S staining.

ALP activity and mineralization analysis. Following culture of cells in osteogenic induction medium for 14 days, rat BMSCs were fixed with $4 \%$ paraformaldehyde at room temperature for $10 \mathrm{~min}$. ALP activity assay was performed using a p-nitrophenyl phosphate assay according to a previously described protocol (26). For mineralization analysis, cells were cultured in osteogenic medium for 21 days. The extent of matrix mineralization was measured by Alizarin red S (Sigma-Aldrich; Merck KGaA) staining which was then quantified using $0.5 \mathrm{~N} \mathrm{HCl}$ and $5 \%$ SDS as previously described (26). The OD was then quantified at $490 \mathrm{~nm}$ using a SpectraMAX M2 microplate reader.

Reverse transcription-quantitative polymerase chain reaction $(R T-q P C R)$. Primary stromal cells were cultured for 10 days as described above. Total RNA was isolated from BMSCs using the TRIzol reagent (Invitrogen; Thermo Fisher Scientific, Inc.) according to the manufacturer's instructions. RT was performed on $0.5 \mathrm{mg}$ total RNA using a Prime Script RT reagent kit with gDNA Eraser (Takara Biotechnology Co., Ltd., Dalian, China). The qPCR assay was performed using the SYBR-Green PCR master mix (Applied Biosystems; Thermo Fisher Scientific, Inc.). The $2^{-\Delta \Delta C q}$ relative quantification method (27) was used to calculate gene expression levels relative to the $\mathrm{CON}$ group. Values were normalized to GAPDH expression. The primer sequences for qPCR are presented in Table I. The reported data represent the mean expression from three experiments.

Statistical analysis. Results are expressed as the mean \pm standard deviation. Statistical analysis was performed using a one-way analysis of variance and Tukey's test for post hoc analysis. $\mathrm{P}<0.05$ was considered to indicate a statistically significant difference.

\section{Results}

MIR has an anabolic effect on trabecular bone. To determine bone adaptation to running at different intensities, the metaphyseal femur was examined by micro-CT. Compared with the Con group, denser and better-organized trabeculae were observed in the MIR group (Fig. 1A-D). Micro-CT analysis of 3D microarchitecture parameters from trabecular bone harvested from the distal rat femora are summarized in Fig. 1E. The results suggested that MIR significantly affected the amount and structural organization of trabecular bone. MIR led to significantly higher BV/TV than in the controls $(\mathrm{P}=0.002)$, indicating a stimulatory effect on trabecular bone mass. Although MIR failed to significantly affect the Tb.Th (thickness) and DA of trabecular bone, MIR significantly decreased the value of Tb.Sp $(\mathrm{P}=0.006)$ and 

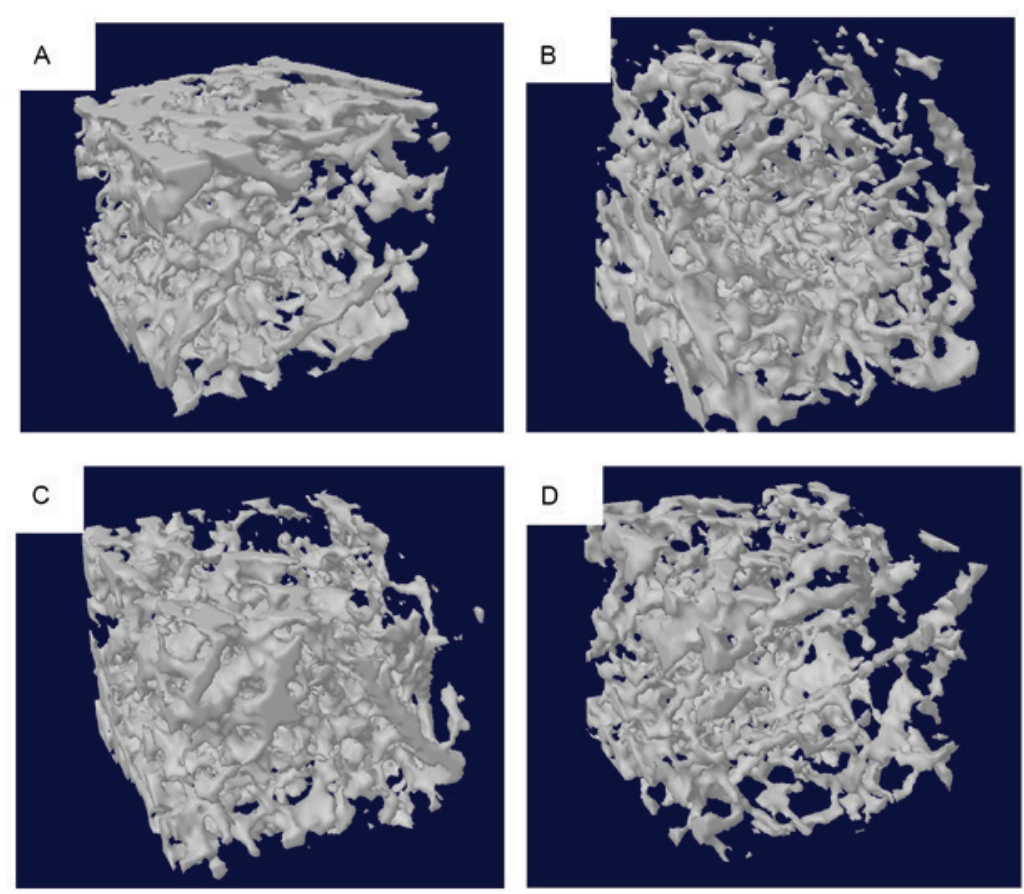

E

\begin{tabular}{lcccc}
\hline & Con & LIR & MIR & HIR \\
\hline BV/TV $(\%)$ & $27.43 \pm 5.03$ & $34.23 \pm 5.44$ & $42.58 \pm 4.81^{*}$ & $30.81 \pm 6.86$ \\
BMD $\left(\mathrm{g} / \mathrm{cm}^{3}\right)$ & $0.265 \pm 0.039$ & $0.310 \pm 0.011$ & $0.299 \pm 0.060$ & $0.275 \pm 0.040$ \\
Tb.N $(1 / \mathrm{mm})$ & $2.428 \pm 0.591$ & $2.758 \pm 0.655$ & $3.013 \pm 0.535$ & $2.609 \pm 0.277$ \\
Tb.Th $(\mathrm{mm})$ & $0.116 \pm 0.013$ & $0.121 \pm 0.006$ & $0.119 \pm 0.009$ & $0.117 \pm 0.007$ \\
Tb.Sp $(\mathrm{mm})$ & $0.441 \pm 0.188$ & $0.293 \pm 0.078$ & $0.227 \pm 0.083^{*}$ & $0.290 \pm 0.094$ \\
SMI & $1.729 \pm 0.380$ & $1.438 \pm 0.342$ & $1.169 \pm 0.594^{*}$ & $1.470 \pm 0.412$ \\
DA & $0.546 \pm 0.025$ & $0.489 \pm 0.056 *$ & $0.509 \pm 0.023$ & $0.516 \pm 0.025$ \\
\hline
\end{tabular}

Figure 1. 3D images of right femoral metaphyseal from (A) Con, (B) LIR, (C) MIR and (D) HIR groups. As compared with Con group, denser and better-organized trabeculae were observed in the MIR group. In addition, microarchitecture parameters of trabecular bone from distal femur in four groups were also presented, and (E) the results demonstrated that MIR significantly affected the amount and structural organization of trabecular bone ( $\mathrm{n}=6$ per group). * $\mathrm{P}<0.05$ vs. Con group. Con, control; LIR, low-intensity running; MIR, moderate-intensity running; HIR, high-intensity running; BV/TV, trabecular bone volume; BMD, bone mineral density; Tb.N, trabecular number; Tb.Th, trabecular thickness; Tb.Sp, trabecular separation; SMI, structure model index; DA, degree of anisotropy.

SMI $(\mathrm{P}=0.043)$ compared with the Con group, suggesting a reduction in trabecular separation with a more plate-like architecture. Thus, the trabeculae became denser following MIR. Notably, there were no obvious changes in either the LIR or HIR groups, except for a significant increase in DA in the LIR group compared with the Con group $(\mathrm{P}=0.012)$. These data indicate that running affects trabecular bone in an intensity-dependent manner, and MIR can enhance trabecular bone mass to improve structural organization.

MIR enhances the osteogenic potential of BMSCs. CFU-F (Fig. 2A) and CFU-Ob (Fig. 2B) assays were performed to elucidate the differentiation potential of BMSCs. It was observed that the number of bone marrow cells that were capable of forming colonies were similar to the CON group for all three running groups (Fig. 2C). This result suggests that, regardless of the intensity, running failed to affect the total number of progenitor cells present. By contrast, in comparison with the Con group, significantly higher numbers of ALP-positive colonies were observed in the MIR group ( $\mathrm{P}=0.001)$, but not in LIR or HIR groups (Fig. 2C), indicating that MIR, but not LIR or HIR, may enhance the osteogenic potential of BMSCs.

MIR enhances the capacity for osteogenic differentiation and maturation of BMSCs. Changes in BMSC proliferation were evaluated using an in vitro colorimetric assay. As compared with untreated Con cells, cell proliferation was significantly enhanced in MIR-treated BMSCs, but not in LIR-or HIR-treated BMSCs (Fig. 3A). In addition, the BMSC ability for osteogenic differentiation was examined by ALP staining and Alizarin red S staining. The number of ALP-positive cells changed in an intensity-dependent manner (Fig. 3B). In 
A
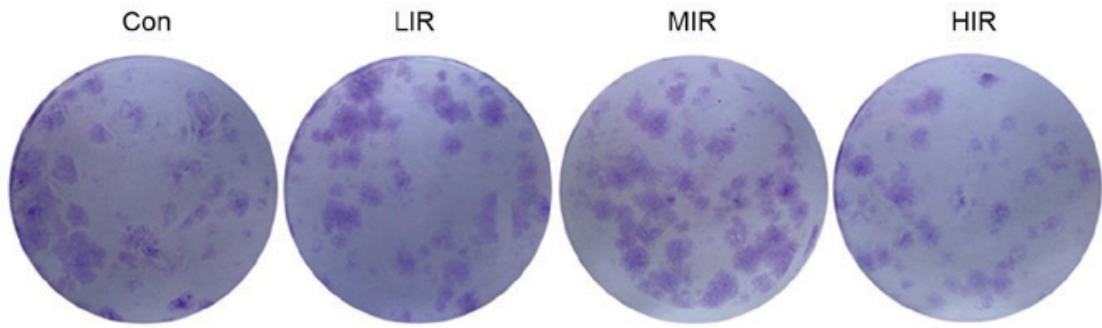

B
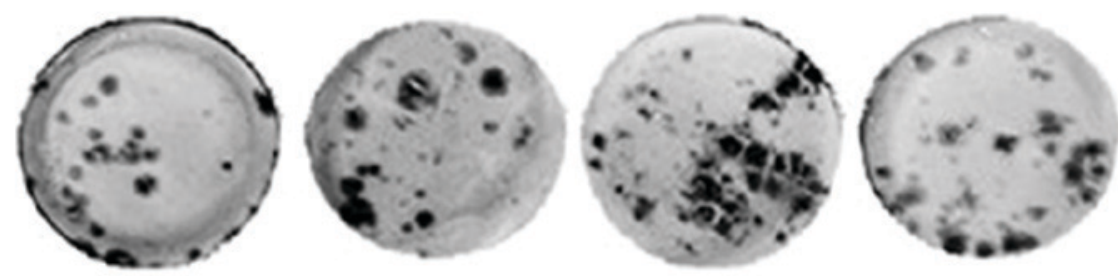

C
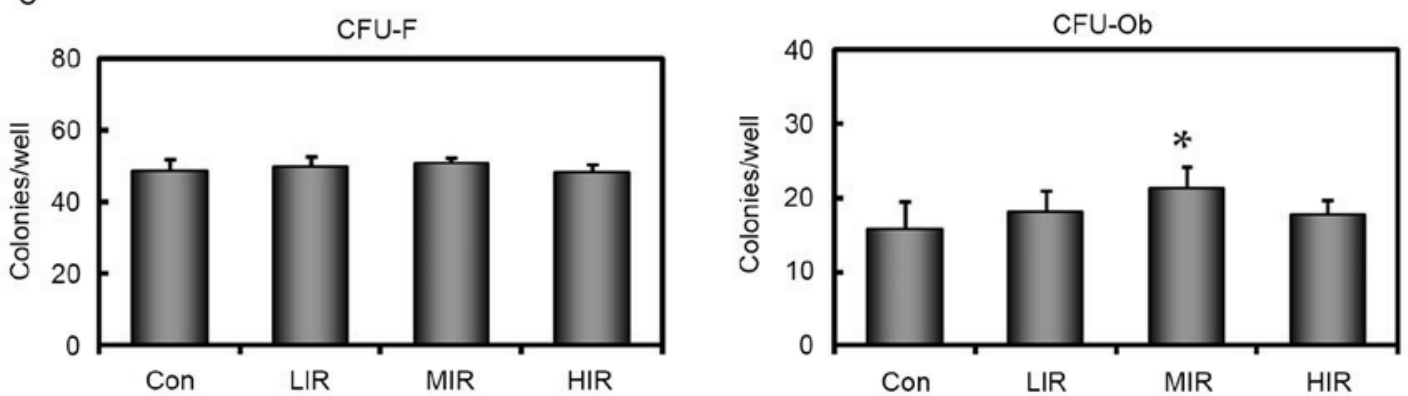

Figure 2. Assays for the number of CFU for fibroblasts and osteoblasts. (A) CFU-F and (B) CFU-Ob assays were performed in duplicate in 6-well plates with $5 \times 10^{5}$ nucleated cells/well. A representative well is presented for each group. (C) Quantitative results for these assays are also presented as bar graphs. In all groups, the number of bone marrow cells capable of forming fibroblast colonies is similar. Compared with the Con group, a significantly higher number of ALP-positive colonies were present in the MIR, but not in LIR and HIR groups ( $\mathrm{n}=6$ per group). ${ }^{\text {" }} \mathrm{P}<0.05$ vs. Con group. Con, control; LIR, low-intensity running; MIR, moderate-intensity running; HIR, high-intensity running; CFU, colony forming units; CFU-F, CFU-fibroblasts; CFU-Ob, CFU-osteoblasts.

a parallel experiment, ALP activity was found to change in a similar manner (Fig. 3B). The most intensive ALP staining and highest ALP activity was observed in MIR-treated BMSCs. A significant difference in ALP activity between MIR-treated and untreated BMSCs was detected $(\mathrm{P}=0.020)$.

To further investigate the terminal differentiation state of BMSCs in each group, cells were cultured in osteogenic induction medium for 21 days and stained with Alizarin red S to visualize mineralized bone nodules. Results demonstrated that BMSCs from all groups formed mineralized nodules in a pattern similar to that seen in ALP staining (Fig. 3C). Mineral content was also quantified in parallel. The most intense ARS staining and the highest mineral content were observed in MIR-treated BMSCs. There was a significant difference in mineral content between the MIR-treated and untreated BMSCs (Fig. 3C). These data suggested that MIR enhanced the capacity for osteogenic differentiation and maturation of BMSCs, whereas, LIR or HIR did not.

MIR upregulates mRNA expression of osteogenic genes. The effect of running on osteogenic differentiation was also investigated by assessing mRNA expression of a number of osteogenesis-associated genes in cultured BMSCs, including RUNX2, SP7, ALP, osteocalcin, and collagen I (Fig. 4). As compared with the Con group, MIR was observed to significantly enhance mRNA expression of all the genes analyzed, which are involved in various stages of osteogenic differentiation of BMSCs. No significant difference in mRNA expression of these genes was detected between LIR and the Con group. Compared with the Con group, HIR produced a significant decrease in mRNA expression of RUNX2 and collagen I, and a significant increase in the mRNA expression of osteocalcin. These results indicated that the effect of running on mRNA expression of osteogenic genes varied with intensity. In addition, MIR may affect various steps of osteogenic differentiation of BMSCs, and upregulate mRNA expression of multiple osteogenic genes.

MIR inhibits the adipogenesis of BMSCs. The marrow adipocyte density was assessed with $\mathrm{H} \& \mathrm{E}$ staining of the femoral diaphyseal section (Fig. 5A-D). Compared with the Con group (34.50 \pm 5.16$)$, a significant decrease in adipocyte density was observed in the MIR group (27.50 \pm 5.00 ;

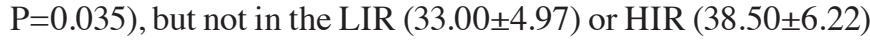
groups (Fig. 5E). Consistent with the intensity-dependent effect on adipocyte density in bone marrow and adipogenic differentiation of isolated BMSCs from treadmill-treated rats, RT-qPCR analyses revealed an intensity-dependent effect on expression of peroxisome proliferator activated receptor $\gamma$

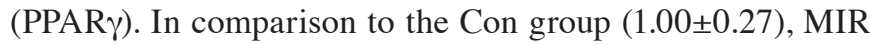
BMSC samples exhibited significantly reduced expression of PPAR $\gamma(0.27 \pm 0.03 ; \mathrm{P}=0.001)$, whereas, LIR $(0.67 \pm 0.10)$ and HIR $(1.05 \pm 0.11)$ were not significantly different to the control (Fig. 5F). These observations suggested that, in addition to the increase in osteogenesis, MIR also induced a reduction in adipogenesis in BMSCs. 
A

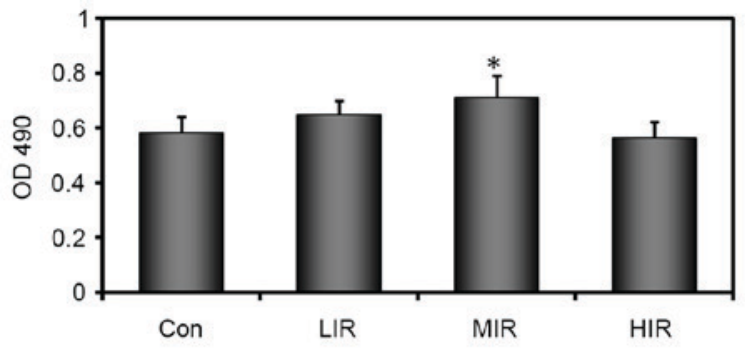

B
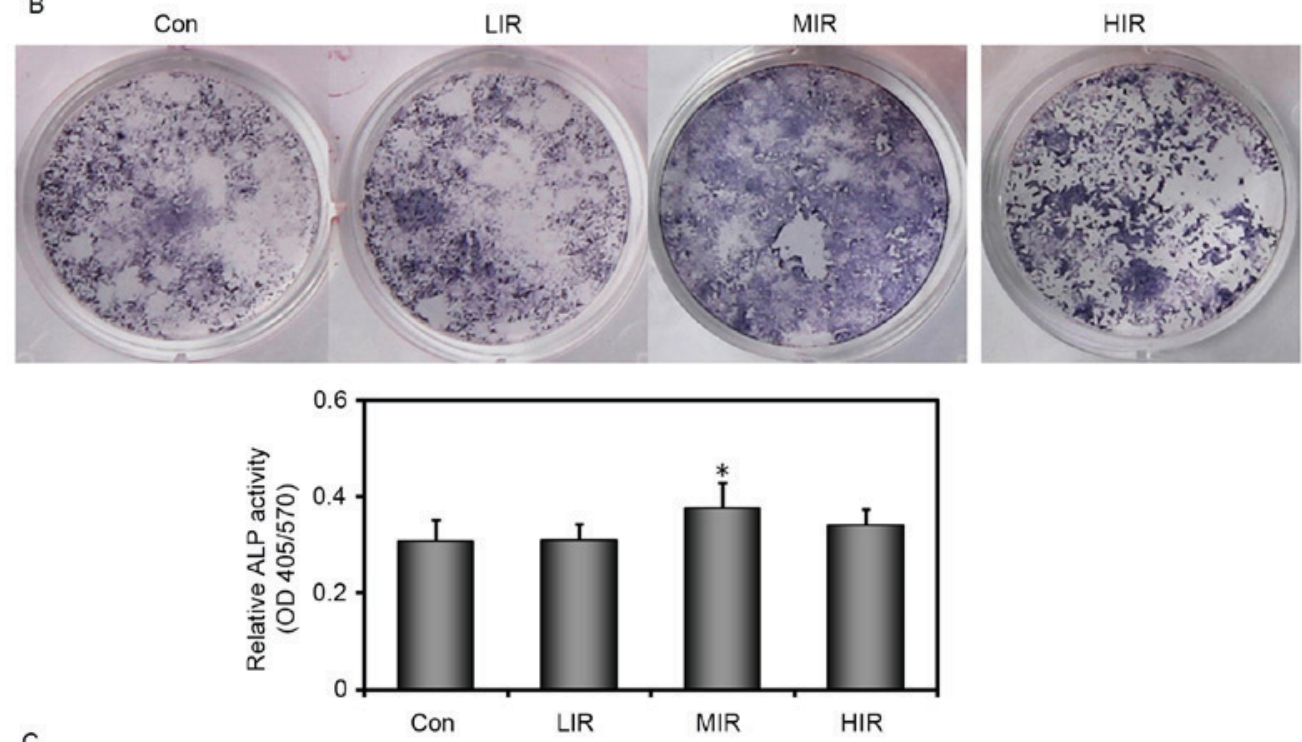

C
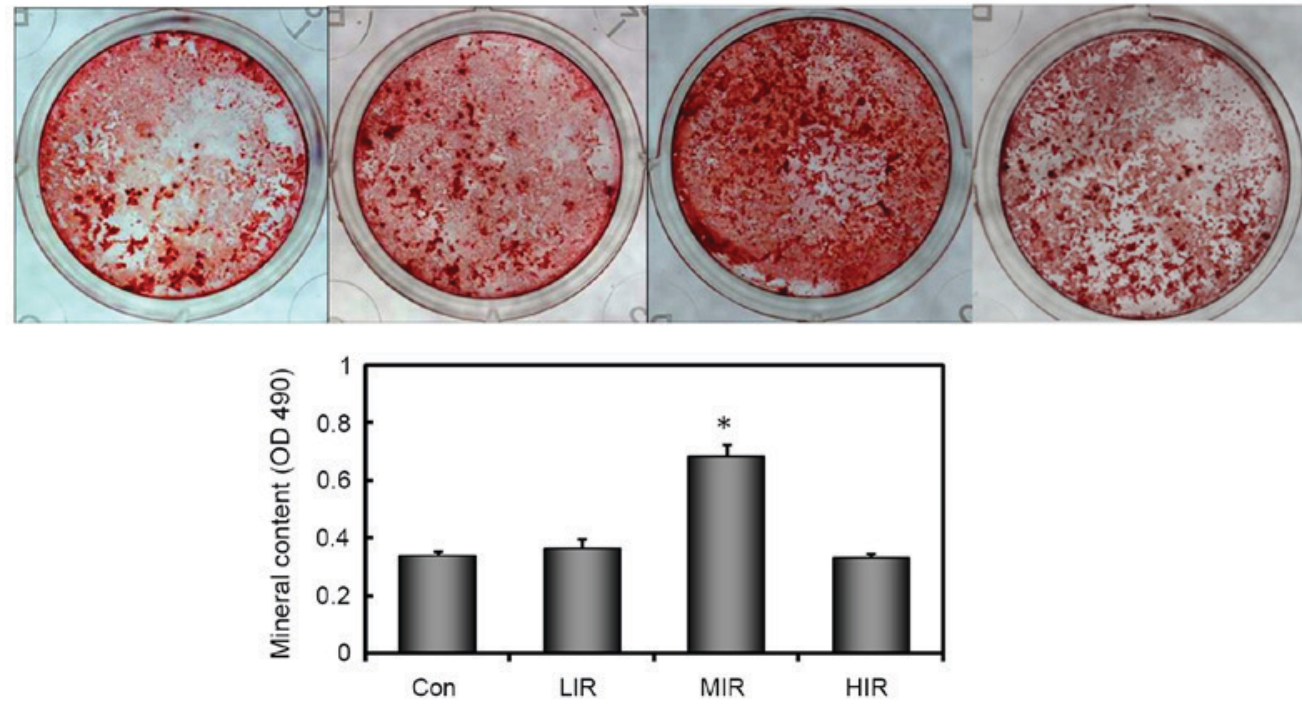

Figure 3. Effect of running on BMSC proliferation and osteogenic differentiation. The rats were either untreated Con, or exposed to LIR, MIR or HIR for 8 weeks. (A) An in vitro colorimetric assay was used to examine cell proliferation. (B) In addition, BMSCs from rats in each group were cultured in osteogenic induction media for 10 days and assayed for ALP activity. (C) Cells were cultured in osteogenic induction medium for 21 days and stained with Alizarin red to visualize mineralized bone nodules ( $\mathrm{n}=6$ per group). " $\mathrm{P}<0.05$ vs. OD, optical density; Con group. Con, control; LIR, low-intensity running; MIR, moderate-intensity running; HIR, high-intensity running; ALP, alkaline phosphatase.

MIR stimulates $\beta$-catenin protein expression in vivo and gene expression in BMSCs. We investigated whether running could enhance the expression of $\beta$-catenin in the femoral diaphysis of rats. There was low $\beta$-catenin staining in sections from the Con group (Fig. 6); however, $\beta$-catenin signals were observed on the cells located in the extracellular matrix (ECM) in the treadmill-treated groups; the most intense and concentrated $\beta$-catenin signals appeared in the MIR group (Fig. 6A-D). The $\beta$-catenin content in each group was quantified, and the results demonstrated a significant increase in MIR (4.46 \pm 0.84 ; $\mathrm{P}<0.001)$, but not in LIR $(2.42 \pm 0.64)$ and HIR groups

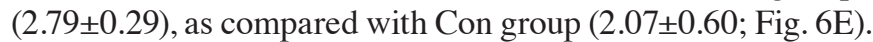
In addition, RT-qPCR was used to analyze the relative mRNA expression of $\beta$-catenin, which is part of the Wnt signaling pathway (25). Compared with the Con group (1.00 \pm 0.13 ), a significant difference in mRNA expression of $\beta$-catenin was observed in MIR $(1.81 \pm 0.17 ; \mathrm{P}=0.001)$, but not in LIR $(1.37 \pm 0.18)$ and HIR groups $(1.36 \pm 0.21$; Fig. $6 \mathrm{~F})$. These data 

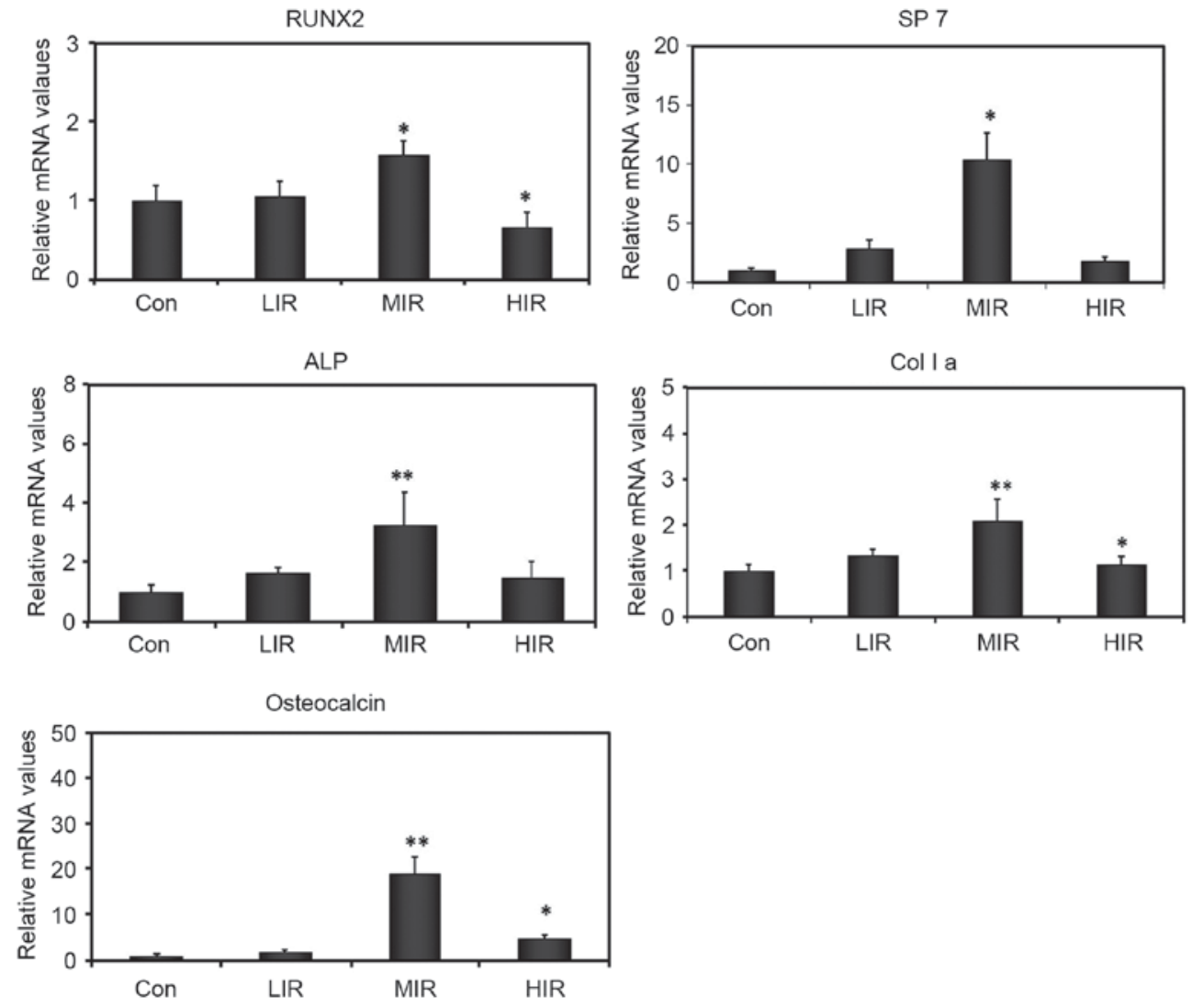

Figure 4. Effect of running on the relative expression of osteogenesis-associated genes in cultured BMSCs analyzed by reverse transcription-quantitative polymerase chain reaction, including RUNX2, SP7, ALP, osteocalcin, and $\mathrm{Col} I \mathrm{I}$ ( $\mathrm{n}=6$ per group). ${ }^{*} \mathrm{P}<0.05$ vs. Con group; ${ }^{* *} \mathrm{P}<0.001$ vs. Con group. Con group. Con, control; LIR, low-intensity running; MIR, moderate-intensity running; HIR, high-intensity running; RUNX2, runt related transcription factor 2; Sp7, Sp7 transcription factor; ALP, alkaline phosphatase; Col I a, collagen I.

demonstrated that MIR stimulates $\beta$-catenin protein expression in vivo and $\beta$-catenin gene expression in BMSCs.

\section{Discussion}

The present study investigated the effects of three different running intensities (LIR, MIR, and HIR) on trabecular bone remodeling using a rat model. The differentiation of BMSCs was also examined under different mechanical conditions to gain insight into the mechanisms responsible for bone homeostasis. By regulating the osteogenic and adipogenic differentiation of the BMSCs, an intensity-dependent effect of running on bone remodeling was noted. This effect may occur by activating the $\mathrm{Wnt} / \beta$-catenin signaling.

Although a number of studies have examined the effect of treadmill running on bone mass in normal and also ovariectomized rats $(3,25,28)$, few studies have attempted to compare variations in running intensity on bone mass. As assessed by micro-CT, the results of the current study indicated that running affects trabecular bone in an intensity-dependent manner. MIR was able to enhance trabecular bone mass with better structural organization compared with either LIR or HIR. Similar intensity-dependent effects from squatting exercises have been reported in an ovariectomized rat model (29). The intensity-dependent effect observed in the present study is likely associated with the different mechanical loads placed on rat femora using the three different running intensity protocols (LIR, MIR and HIR).
In the LIR group, no beneficial effect was observed on bone mass, which is consistent with a number of human and animal studies $(30,31)$. Animal studies have reported that the induced mechanical strain must reach a 'set point' threshold before an osteogenic effect is initiated. Once this threshold is exceeded, bone formation is positively correlated with peak strain magnitude $(32,33)$. In this study, LIR may have induced a mechanical strain that was below the 'set point' threshold, whereas, the induced mechanical strain by MIR exceeded the threshold. As excessive loading, or prolonged unloading, may have detrimental effects on bone (10), the finding that HIR had little effect on either bone amount or structure in the present study suggests that HIR may induce a mechanical load close to the microdamage threshold.

Notably, using the same animal model, we previously demonstrated that running with low-to-moderate intensity can maintain the integrity of cartilage, whereas, high-intensity running damages articular cartilage (24). Similar to cartilage, there is likely a biomechanical 'window' that is required to maintain optimal bone homeostasis. The running-induced strain signals within this biomechanical 'window' could result in proliferation and osteogenic differentiation of BMSCs. This may partially explain the observation of intensity-dependent bone adaptation in rat femora.

Bone has a remarkable ability to adjust its mass and architecture in response to mechanical loading, which may be associated with the ability of bone cells to sense tiny strains within the bone matrix. The effect of mechanical stimuli 

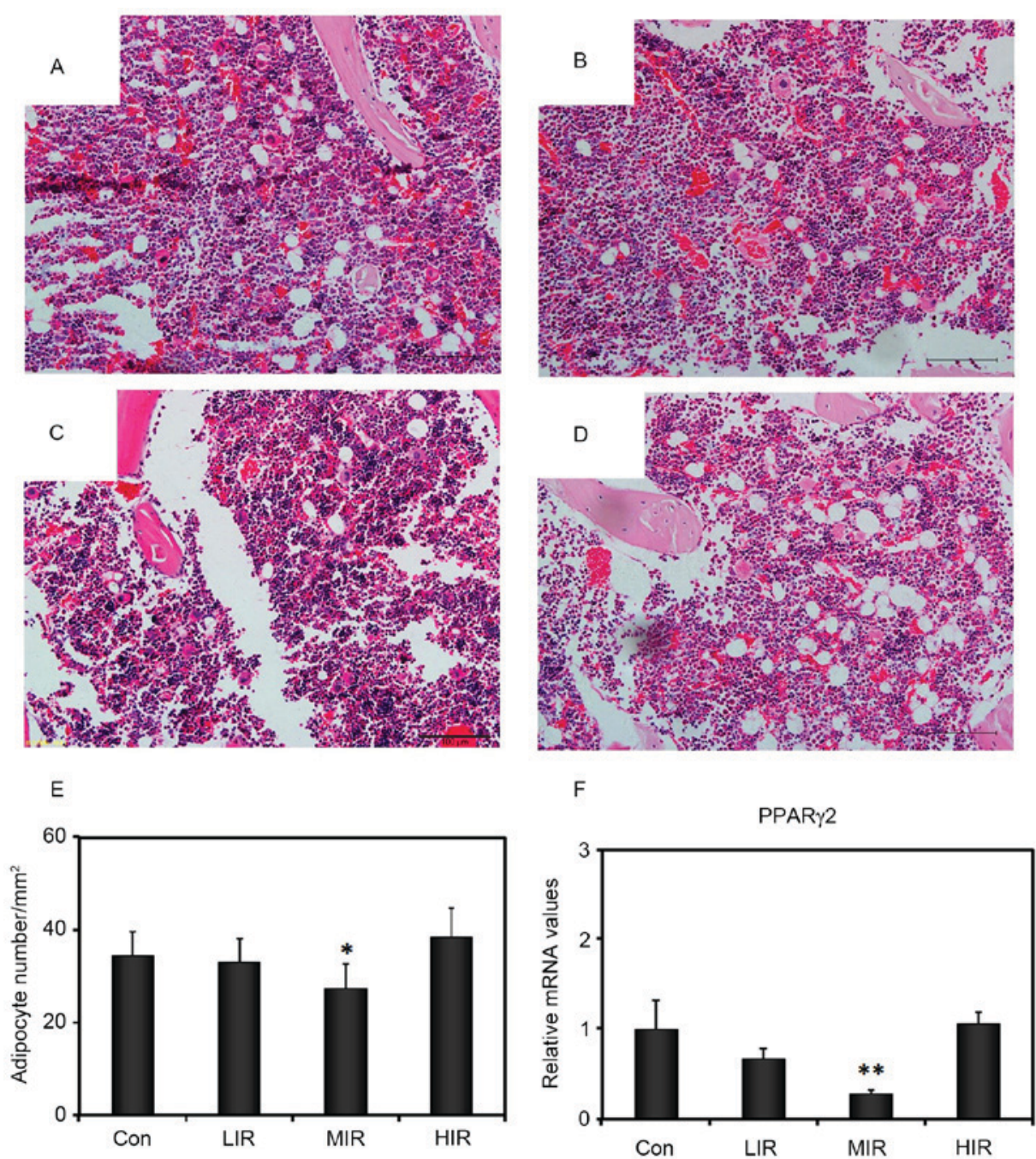

Figure 5. Effect of running on bone marrow adipocyte density and expression of PPAR $\gamma$ in cultured BMSCs. Hematoxylin and eosin staining of the femoral diaphyseal sections in (A) Con, (B) LIR, (C) MIR and (D) HIR groups (scale bar, $100 \mathrm{~mm}$ ). (E) The marrow adipocyte density was quantified in the four groups . (F) Expression of PPAR $\gamma 2$ was determined by reverse transcription-quantitative polymerase chain reaction ( $n=6$ per group). ${ }^{*} \mathrm{P}<0.05$ vs. Con; ${ }^{* * *} \mathrm{P}<0.001 \mathrm{vs}$. Con group. Con, control; LIR, low-intensity running; MIR, moderate-intensity running; HIR, high-intensity running; PPAR $\gamma 2$, peroxisome proliferator activated receptor $\gamma 2$.

on bone cell metabolism may be modulated by a number of variables, such as the type of bone cell (34) and the amount of mechanical stimuli (35). It appears that the magnitude of the load has a crucial role in the response of bone cells to mechanical loading. Jagodzinski et al (36) demonstrated that $8 \%$ cyclic stretching in silicone-dishes significantly increased the secretion of differentiation markers by both osteoblasts and BMSCs compared with either $2 \%$ cyclic stretching or no stimulation. Bacabac et al (34) overstimulated bone cells with noisy fluid shear stress and suggested that noise enhances the molecular response through a threshold-activated mechanism. Our results indicated that mechanical strain must reach a 'set point' threshold before the proliferation and osteogenic differentiation of BMSCs can be activated. On the other hand, too much strain can induce microdamage in the matrix and exacerbate the death of cells adjacent to the damaged matrix (37). Excessive cycles may have an inhibitory (rather than a stimulatory) effect on osteoblast proliferation (38). The results of the current study suggested that HIR may exert too much strain, which may fail to activate (or even suppress) the proliferation and osteogenic differentiation of BMSCs.
In the present study, the proliferation and osteogenic differentiation potential of BMSCs were investigated in an attempt to delineate the underlying mechanism responsible for the adaptation of trabecular bone to treadmill running at different intensities. From the results of MTT assays, MIR was demonstrated to stimulate the proliferation of BMSCs, whereas, LIR or HIR did not. The results also revealed that running did not induce alteration in CFU-F. Similar results were previously reported in rats following climbing exercises (22) and unloading (39), indicating that mechanical loading had no impact on the total number of progenitor cells present. By contrast, MIR was found to increase CFU-Ob, while LIR and HIR did not. It was reported previously that climbing exercises (22) and unloading (39) may lead to an increase and decrease in CFU-Ob, respectively. As such, there is likely a biomechanical 'window', in which the running-induced strain signals increase the number of BMSCs and progenitor cells specific to the osteoblast lineage.

The results of the current also suggested that running affects the osteogenic differentiation of BMSCs in an intensity-dependent manner. Osteoblastic differentiation is a 

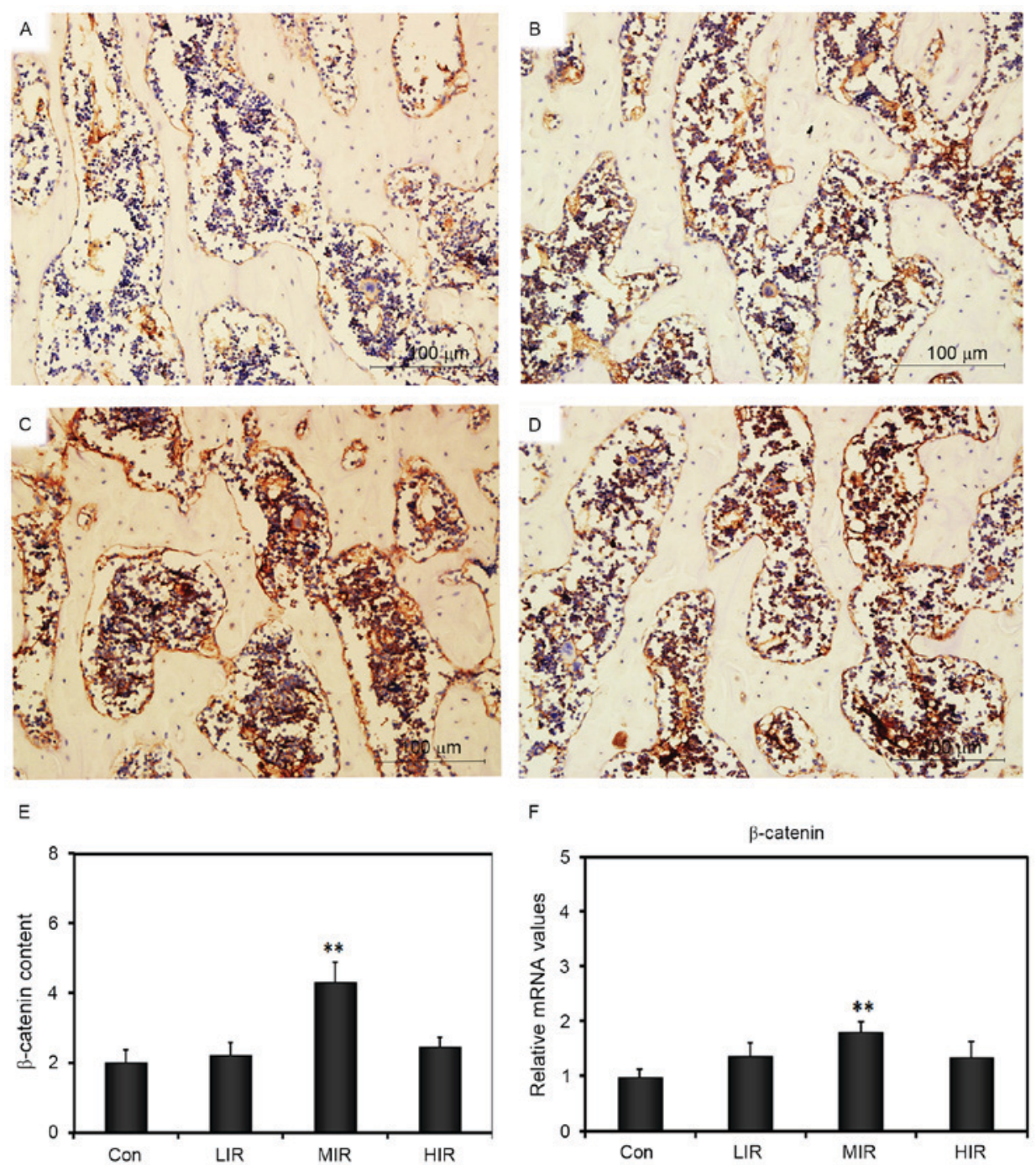

Figure 6. Protein expression of $\beta$-catenin in femoral diaphyseal bone as detected by immunohistochemistry and the gene expression of $\beta$-catenin cultured BMSCs analyzed by reverse transcription-quantitative polymerase chain reaction. $\beta$-catenin staining in the (A) Con, (B) LIR, (C) MIR and (D) HIR groups. (E) The $\beta$-catenin content from staining of each group was quantified using Image-Pro-Plus. (F) mRNA expression of $\beta$-catenin (n=6 per group). ** $\mathrm{P}<0.001 \mathrm{vs.}$ Con group. Con, control; LIR, low-intensity running; MIR, moderate-intensity running; HIR, high-intensity running.

complex process that involves differentiation of mesenchymal cells into pre-osteoblasts and osteoblasts, ultimately leading to synthesis and deposition of bone matrix proteins. This process is initially driven by RUNX2, and then by SP7. It is characterized by expression of ALP and osteocalcin, and mineralization of the ECM (21). RUNX2 is a key transcription factor required for osteoblast differentiation. SP7 is a late bone marker required for differentiation of pre-osteoblasts into functioning osteoblasts (40). ALP is a marker of early osteoblast differentiation, associated with synthesis of organic bone matrix before mineralization of the organic bone matrix. Collagen I is the most abundant protein in the bone ECM with a high level of expression in osteoblasts. The expression of collagen I gene occurs at all stages during bone development (41). Osteocalcin is expressed by mature osteoblasts in association with matrix mineralization (42). The increased mRNA expression of these transcription factors, together with the enhanced capacity for osteogenic differentiation and maturation of BMSCs (as reflected by ALP activity and Alizarin red S staining), indicated that MIR may stimulate the osteoblastic differentiation of BMSCs at various stages of this process. However, no significant change in mRNA expression of these transcription factors (nor in osteogenic differentiation and maturation of BMSCs) was observed in the LIR group. A significant decrease in mRNA expression of RUNX2 and collagen I was observed in the HIR group, with a significant increase in osteocalcin mRNA expression.

In addition to osteogenic differentiation, running also affects the adipogenic differentiation of BMSCs in an intensity-dependent manner. MIR led to a dramatic decrease in adipocyte density and expression of PPAR $\gamma$, whereas, LIR or HIR did not. As adipocytes and osteoblasts share a common stromal precursor cell pool in the bone marrow, the inverse relationship between osteogenic and adipogenic differentiation of BMSCs has been extensively studied $(12,16,20-22)$. Consistent with these reports, the results of the current study demonstrated that MIR induces a decrease in marrow adiposity, which accompanied an observed increase in overall bone volume. In support of the histological increase in marrow adiposity, MIR-treated BMSCs had a significantly lower adipogenic potential, suggesting that MIR treatment alters the microenvironment to support bone formation and inhibit fat 
formation within the bone marrow. Concordantly, expression of PPAR $\gamma$ in cultured BMSCs decreased. PPAR $\gamma$ causes bone loss in animals and humans, at least in part due to the suppression of osteoblast differentiation from BMSCs $(21,43,44)$. Previously, treadmill running with moderate intensity led to positive inhibition effect on bone marrow adipogenesis and expression of PPAR $\gamma$ in ovariectomized rats (28). The in vivo findings of the present study are supported by the in vitro observation that MIR-treated BMSCs exhibited a decrease in adipogenic differentiation potential, illustrating that MIR may directly inhibit adipogenic commitment of BMSCs.

Mechanical loading is crucial for bone cells to adjust bone architecture in order to maintain bone strength (45). It is believed that the $\mathrm{Wnt} / \beta$-catenin signaling pathway is involved in this response, since $\mathrm{Wnt} / \beta$-catenin signaling is not only a normal physiological response of bone to mechanical loading $(46,47)$, but is also a component of the early response of osteoblastic bone cells to load-bearing (44). In the current study, the enhancement of protein and gene expression of $\beta$-catenin induced by MIR may have mediated the activation of transcription factors such as RUNX2 (16), and the downregulation of PPAR $\gamma$ (48), thus biasing BMSC differentiation away from adipogenesis and towards osteoblastogenesis (49). The results of the present study suggested that the effect of MIR on decreasing fat mass and increasing bone mass may function through the regulation of BMSC differentiation via inhibiting PPAR $\gamma$ and stimulating $\beta$-catenin. Since the level of $\beta$-catenin is an indication of the $\mathrm{Wnt} / \beta$-catenin signaling activation (50), the results support an involvement of the $\mathrm{Wnt} / \beta$-catenin signaling pathway in MIR-induced increase in bone mass in rats.

In conclusion, the present study demonstrated that treadmill running appears to affect trabecular bone mass in an intensity-dependent manner. In addition, a biomechanical 'window' may exist that maintains optimal bone homeostasis. By regulating the osteogenic and adipogenic differentiation of the BMSCs, an intensity-dependent effect of running on bone remodeling was observed. This result may provide insight into the molecular and cellular mechanisms responsible for bone adaptation by activating the $\mathrm{Wnt} / \beta$-catenin signaling.

\section{Acknowledgements}

We gratefully acknowledge Mr. PR Zhaoat from the Key Laboratory of Bone and Cartilage Regenerative Medicine in Southern Medical University (Guangdong, China) for his technical assistance. We also thank Professor Allen P. Liangat from the Department of Orthopedics and Traumatology, Nanfang Hospital, Southern Medical University for revision of this manuscript.

\section{Funding}

This study was supported by National Natural Science Foundation of China (grant no. 81572219) and Guangdong Natural Science Foundation (grant no. 2014A030313307).

\section{Availability of data and materials}

All data generated or analyzed during this study are included in this published article.

\section{Authors' contributions}

GXN and MY designed the study. SYL, ZL, and SYX performed the experiments. SYL and ZL collected the data. SYL and LX analyzed the data. SYL, ZL, and SYX interpreted the data. GXN, MY, SYL, ZL, SYX, and LX wrote the manuscript. All authors read and approved the final manuscript.

\section{Ethics approval and consent to participate}

This study was approved by the animal ethics committee of Nanfang Hospital, Southern Medical University (application no. NFYY-2013-26).

\section{Consent for publication}

Not applicable.

\section{Competing interests}

The authors declare that they have no competing interests.

\section{References}

1. Williams PT: Lower prevalence of hypertension, hypercholesterolemia, and diabetes in marathoners. Med Sci Sports Exerc 41: 523-529, 2009.

2. Schneider S, Askew CD, Diehl J, Mierau A, Kleinert J, Abel T, Carnahan $\mathrm{H}$ and Strüder HK: EEG activity and mood in health orientated runners after different exercise intensities. Physiol Behav 96: 709-716, 2009.

3. Iwamoto J, Takeda T and Sato Y: Effect of treadmill exercise on bone mass in female rats. Exp Anim 54: 1-6, 2005.

4. Bourrin S, Genty C, Palle S, Gharib C and Alexandre C: Adverse effects of strenuous exercise: a densitometric and histomorphometric study in the rat. J Appl Physiol (1985) 76: 1999-2005, 1994.

5. Hind K, Truscott JG and Evans JA: Low lumbar spine bone mineral density in both male and female endurance runners. Bone 39: 880-885, 2006.

6. Lappe J, Cullen D, Haynatzki G, Recker R, Ahlf R and Thompson K: Calcium and vitamin d supplementation decreases incidence of stress fractures in female navy recruits. J Bone Miner Res 23: 741-749, 2008.

7. Frost HM: Bone's mechanostat: A 2003 update. Anat Rec A Discov Mol Cell Evol Biol 275: 1081-1101, 2003.

8. Ozcivici E, Luu YK, Adler B, Qin YX, Rubin J, Judex S and Rubin CT: Mechanical signals as anabolic agents in bone. Nat Rev Rheumatol 6: 50-59, 2010.

9. Rubin CT and Lanyon LE: Regulation of bone mass by mechanical strain magnitude. Calcif Tissue Int 37: 411-417, 1985.

10. Scott A, Khan KM, Duronio V and HartDA: Mechanotransduction in human bone: In vitro cellular physiology that underpins bone changes with exercise. Sports Med 38: 139-160, 2008.

11. Klein-Nulend J, Bacabac RG and Bakker AD: Mechanical loading and how it affects bone cells: The role of the osteocyte cytoskeleton in maintaining our skeleton. Eur Cell Mater 24: 278-291, 2012.

12. Georgiou KR, Scherer MA, Fan CM, Cool JC, King TJ, Foster BK and Xian CJ: Methotrexate chemotherapy reduces osteogenesis but increases adipogenic potential in the bone marrow. J Cell Physiol 227: 909-918, 2012.

13. Bianco P and Gehron Robey P: Marrow stromal stem cells. J Clin Invest 105: 1663-1668, 2000.

14. Kajkenova O,Lecka-Czernik B, Gubrij I, Hauser SP, Takahashi K, Parfitt AM, Jilka RL, Manolagas SC and Lipschitz DA: Increased adipogenesis and myelopoiesis in the bone marrow of SAMP6, a murine model of defective osteoblastogenesis and low turnover osteopenia. J Bone Miner Res 12: 1772-1779, 1997.

15. Nuttall ME and Gimble JM: Is there a therapeutic opportunity to either prevent or treat osteopenic disorders by inhibiting marrow adipogenesis? Bone 27: 177-184, 2000. 
16. Nuttall ME and Gimble JM: Controlling the balance between osteoblastogenesis and adipogenesis and the consequent therapeutic implications. Curr Opin Pharmacol 4: 290-294, 2004.

17. Tsai MY, Shyr CR, Kang HY, Chang YC, Weng PL, Wang SY, Huang KE and Chang C: The reduced trabecular bone mass of adult ARKO male mice results from the decreased osteogenic differentiation of bone marrow stroma cells. Biochem Biophys Res Commun 411: 477-482, 2011.

18. Mauney JR, Sjostorm S, Blumberg J, Horan R, O'Leary JP, Vunjak-Novakovic G, Volloch V and Kaplan DL: Mechanical stimulation promotes osteogenic differentiation of human bone marrow stromal cells on 3-D partially demineralized bone scaffolds in vitro. Calcif Tissue Int 74: 458-468, 2004.

19. Sakata T, Sakai A, Tsurukami H, Okimoto N, Okazaki Y, Ikeda S, Norimura T and Nakamura T: Trabecular bone turnover and bone marrow cell development in tail-suspended mice. J Bone Miner Res 14: 1596-1604, 1999.

20. Ahdjoudj S, Kaabeche K, Holy X, Fromigué O, Modrowski D, Zérath E and Marie PJ: Transforming growth factor-beta inhibits CCAAT/enhancer-binding protein expression and PPARgamma activity in unloaded bone marrow stromal cells. Exp Cell Res 303: 138-147, 2005.

21. David V, Martin A, Lafage-Proust MH, Malaval L, Peyroche S, Jones DB, Vico L and Guignandon A: Mechanical loading down-regulates peroxisome proliferator-activated receptor gamma in bone marrow stromal cells and favors osteoblastogenesis at the expense of adipogenesis. Endocrinology 148: 2553-2562, 2007.

22. Mori T, Okimoto N, Sakai A, Okazaki Y, Nakura N, Notomi T and Nakamura T: Climbing exercise increases bone mass and trabecular bone turnover through transient regulation of marrow osteogenic and osteoclastogenic potentials in mice. J Bone Miner Res 18: 2002-2009, 2003 .

23. Yokota H, Leong DJ and Sun HB: Mechanical loading: Bone remodeling and cartilage maintenance. Curr Osteoporos Rep 9: 237-242, 2011

24. Ni GX, Liu SY, Lei L, Li Z, Zhou YZ and Zhan LQ Intensity-dependent effect of treadmill running on knee articular cartilage in a rat model. Biomed Res Int 2013: 172392, 2013.

25. Bu S, Chen Y, Wang S, Zhang F and Ji G: Treadmill training regulates $\beta$-catenin signaling through phosphorylation of GSK-3 $\beta$ in lumbar vertebrae of ovariectomized rats. Eur J Appl Physiol 112: 3295-3304, 2012.

26. Su N, Sun Q, Li C, Lu X, Qi H, Chen S, Yang J, Du X, Zhao L, $\mathrm{He} \mathrm{Q}$, et al: Gain-of-function mutation in FGFR3 in mice leads to decreased bone mass by affecting both osteoblastogenesis and osteoclastogenesis. Hum Mol Genet 19: 1199-1210, 2010.

27. Livak KJ and Schmittgen TD: Analysis of relative gene expression data using real-time quantitative PCR and the 2(-Delta DeltaC(T)) method. Methods 25: 402-408, 2001.

28. Chen Y, Wang S, Bu S, Wang Y, Duan Y and Yang S: Treadmill training prevents bone loss by inhibition of PPAR $\gamma$ expression but not promoting of Runx2 expression in ovariectomized rats. Eur J Appl Physiol 111: 1759-1767, 2011.

29. Kiuchi A, Shimegi S, Tanaka I, Izumo N, Fukuyama R, Nakamuta $\mathrm{H}$ and Koida M: Dose-response effects of exercise intensity on bone in ovariectomized rats. Int J Sports Health Sci 4: 10-18, 2006

30. Huang TH, Lin SC, Chang FL, Hsieh SS, Liu SH and Yang RS: Effects of different exercise modes on mineralization, structure, and biomechanical properties of growing bone. J Appl Physiol (1985) 95: 300-307, 2003

31. Sööt T, Jürimäe T, Jürimäe J, Gapeyeva $H$ and Pääsuke $M$ : Relationship between leg bone mineral values and muscle strength in women with different physical activity. J Bone Miner Metab 23: 401-406, 2005.

32. Turner CH, Forwood MR, Rho JY and Yoshikawa T: Mechanical loading thresholds for lamellar and woven bone formation. J Bone Miner Res 9: 87-97, 1994.

33. Chow JW, Jagger CJ and Chambers TJ: Characterization of osteogenic response to mechanical stimulation in cancellous bone of rat caudal vertebrae. Am J Physiol 265: E340-E347, 1993.
34. Bacabac RG, Van Loon JJ, Smit TH and Klein-Nulend J: Noise enhances the rapid nitric oxide production by bone cells in response to fluid shear stress. Technol Health Care 17: 57-65, 2009.

35. Li YJ, Batra NN, You L, Meier SC, Coe IA, Yellowley CE and Jacobs CR: Oscillatory fluid flow affects human marrow stromal cell proliferation and differentiation. J Orthop Res 22: 1283-1289, 2004.

36. Jagodzinski M, Drescher M,Zeichen J, Hankemeier S, Krettek C, Bosch U and van Griensven M: Effects of cyclic longitudinal mechanical strain and dexamethasone on osteogenic differentiation of human bone marrow stromal cells. Eur Cell Mater 7: 35-41, 2004.

37. Verborgt O, Gibson GJ and Schaffler MB: Loss of osteocyte integrity in association with microdamage and bone remodeling after fatigue in vivo. J Bone Miner Res 15: 60-67, 2000.

38. Stanford CM, Welsch F, Kastner N, Thomas G, Zaharias R, Holtman K and Brand RA: Primary human bone cultures from older patients do not respond at continuum levels of in vivo strain magnitudes. J Biomech 33: 63-71, 2000.

39. Basso N, Jia Y, Bellows CG and Heersche JN: The effect of reloading on bone volume, osteoblast number, and osteoprogenitor characteristics: Studies in hind limb unloaded rats. Bone 37: 370-378, 2005

40. Cao Y, Zhou Z, de Crombrugghe B, Nakashima K, Guan H, Duan X, Jia SF and Kleinerman ES: Osterix, a transcription factor for osteoblast differentiation, mediates antitumor activity in murine osteosarcoma. Cancer Res 65: 1124-1128, 2005.

41. Jikko A, Harris SE, Chen D, Mendrick DL and Damsky CH: Collagen integrin receptors regulate early osteoblast differentiation induced by BMP-2. J Bone Miner Res 14: 1075-1083, 1999

42. Pockwinse SM, Wilming LG, Conlon DM, Stein GS and Lian JB: Expression of cell growth and bone specific genes at single cell resolution during development of bone tissue-like organization in primary osteoblast cultures. J Cell Biochem 49: 310-323, 1992.

43. Shockley KR, Lazarenko OP, Czernik PJ, Rosen CJ, Churchill GA and Lecka-Czernik B: PPARgamma2 nuclear receptor controls multiple regulatory pathways of osteoblast differentiation from marrow mesenchymal stem cells. J Cell Biochem 106: 232-246, 2009.

44. Bruedigam C, Koedam M, Chiba H,Eijken M and van Leeuwen JP: Evidence for multiple peroxisome proliferator-activated receptor gamma transcripts in bone: Fine-tuning by hormonal regulation and mRNA stability. FEBS Lett 582: 1618-1624, 2008

45. Armstrong VJ, Muzylak M, Sunters A, Zaman G, Saxon LK, Price JS and Lanyon LE: Wnt/beta-catenin signaling is a component of osteoblastic bone cell early responses to load-bearing and requires estrogen receptor alpha. J Biol Chem 282: 20715-20727, 2007.

46. Bonewald LF and Johnson ML: Osteocytes, mechanosensing and Wnt signaling. Bone 42: 606-615, 2008.

47. Robinson JA, Chatterjee-Kishore M, Yaworsky PJ, Cullen DM, Zhao W, Li C, Kharode Y, Sauter L, Babij P, Brown EL, et al: Wnt/beta-catenin signaling is a normal physiological response to mechanical loading in bone. J Biol Chem 281: 31720-31728, 2006.

48. Takada I, Kouzmenko AP and Kato S: Wnt and PPARgamma signaling in osteoblastogenesis and adipogenesis. Nat Rev Rheumatol 5: 442-447, 2009.

49. Sen B, XieZ, Case N, Ma M, Rubin C and Rubin J: Mechanical strain inhibits adipogenesis in mesenchymal stem cells by stimulating a durable beta-catenin signal. Endocrinology 149: 6065-6075, 2008.

50. Case N, Ma M, Sen B, Xie Z, Gross TS and Rubin J: Beta-catenin levels influence rapid mechanical responses in osteoblasts. J Biol Chem 283: 29196-29205, 2008.

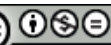

This work is licensed under a Creative Commons Attribution-NonCommercial-NoDerivatives 4.0 International (CC BY-NC-ND 4.0) License. 